\title{
Changing Trends In Acute Pancreatitis -A Retrospective Study
}

\author{
Dr. V.S Venkadesan ${ }^{1}$, Dr. D.N Renganathan ${ }^{2}$ \\ ${ }^{1}$ (Department Of General Surgery, Coimbatore Medical College and Hospital, India) \\ ${ }_{2}^{2}$ (Department Of General Surgery, Coimbatore Medical College and Hospital, India)
}

\begin{abstract}
:
Aim: To analyse the role of alcohol as a causative agent of acute pancreatitis in patients getting admitted at $\mathrm{CMCH}$ with features of acute pancreatitis were enrolled in study

Methods:The patients admitted with features of acute pancreatitis were examined clinically, basic investigations was done and diagnosis was confirmed with the investigations like serum amylase, USG abdomen and pelvis , CECT abdomen and pelvis. Retrospectively history of number of years of alcohol intake was elicited and also the severity of pancreatitis was assessed.

Results: it was observed that major cause of acute pancreatitis was found to be alcohol intake and the incidence of acute severe pancreatitis has also reduced very much.

Conclusion: Alcohol is the major causative factor for acute pancreatitis and the incidence of severe forms of pancreatitis has reduced in the modern era especially over the past decade.
\end{abstract}

Keywords : acute pancreatitis, alcohol, severity

\section{Introduction}

Acute pancreatitis is a commonly occurring surgical emergency and it is diagnosed based on clinical examination and others blood investigations like serum amylase and imaging modalities like USG abdomen and pelvis, CECT abdomen and pelvis. The pathogenesis of acute pancreatitis is the inflammation of pancreatic tissue and inappropriate activation of pancreatic enzymes. The major causes of acute pancreatitis are gall stones , alcohol intake, autoimmune , infections , metabolic disorders , penetrating injuries , abdominal trauma , etc.. The surgeons aim is to identify the cause of acute pancreatitis and to eliminate the cause so that another episode doesn't occur, eliminating the cause is the major line of management of acute pancreatitis. The treatment varies according to the severity, so grading of severity is also very important in the line of management.

\section{Background and purpose of the study:}

There is a changing trend in the cause of acute pancreatitis and also patient presents with less severe variety of acute pancreatitis. In this study we would like to focus the most common cause of acute pancreatitis in our set up and also to find out the severity with which the patients commonly presents. The cause is identified with the retrospective history from the patient and the severity is analysed from the ransons score of severity.

\section{Changing trend in causes of acute pancreatitis :}

The incidence of acute pancreatitis (AP) has increased during the past 20 years. AP is responsible for more than 300,000 hospital admissions annually in the United States. Most patients develop a mild and selflimited course; however, $10 \%$ to $20 \%$ of patients have a rapidly progressive inflammatory response associated with prolonged length of hospital stay and significant morbidity and mortality. Patients with mild pancreatitis have a mortality rate of less than $1 \%$ but, in severe pancreatitis, these increases up to $10 \%$ to $30 \%$.

Gallstone pancreatitis is the most common cause of AP in the West. It accounts for $40 \%$ of U.S. cases. Excessive ethanol consumption is the second most common cause of AP worldwide. But in our community we find a very less percentage of people with acute pancreatitis having gall stones as a predisposing factor unlike the west . We also felt that there is a overall decrease in the severity of the acute pancreatitis and the number of days of hospital stay.

Study Area :

\section{Materials And Methods}

\section{Coimbatore Medical College Hospital [CMCH] , Coimbatore.}

Study population : Patients admitted in $\mathrm{CMCH}$ with symptoms suggestive of acute pancreatitis. Inclusion criteria:

1. Patients presenting with features of acute pancreatitis and confirmed by investigations.

2. Patients older than 12 years of age. 


\section{Exclusion criteria:}

1. Patients not willing to participate in the study (who refused to consent).

2. Patients with other coexisting acute inflammatory conditions

Study Period:

4 years From January 2013- December 2016

Sample Size: 50.All patients eligible by inclusion and exclusion criteria are to be included in the study.

Study Design: A Retrospective study is to be conducted on patients admitted in $\mathrm{CMCH}$ with features of acute pancreatitis and confirmed by investigations . Informed consent will be taken from each respondent.

\section{Study Tools:}

I. Detailed history

II. Serum amylase

III. USG abdomen and pelvis

IV. CECT abdomen and pelvis

\section{Parameters to be studied:}

Preformed Performa which includes the demographic profile of each patient and duration of symptoms like fever, vomiting, abdominal pain, constipation, history of number of years of intake of alcohol Laboratory investigations like complete blood count, serum electrolytes, serum creatinine, blood urea, blood sugar, serum calcium are to be done

Imaging modalities includes USG abdomen and pelvis, CECT abdomen and pelvis .

\section{Methodology:}

Patients admitted in Coimbatore Medical college Hospital with clinical examination suggestive of acute pancreatitis and confirmed with various laboratory investigations and imaging modalities were enrolled for this study after informed consent. Retrospective history was obtained from the patient and the history of number of years of alcohol intake was obtained from the patient. The severity of the disease was also assessed by means of ransons severity index .

\section{Plan for analysis of data:}

Data is to be analyzed using the statistical package SPSS 17. Chi-square tests, student's t tests, significance testing, and $95 \%$ confidence interval formulation are to be carried out wherever appropriate.

\section{Discussion}

Of 50 patients included in the study 44 were more than 30 years of age and 6 were less than 30 years of age with the age of the oldest person being 63 years. There were 47 males and 03 females. About 42 patients had symptoms less than 2 days duration while 8 of them had symptoms for more than 2 days. On Classifying according to dietary habit, there were 10 vegetarians and 40 non vegetarians. The laboratory investigations show all the patients included in the study had more than 3 fold rise in serum amylase level and diagnosis was confirmed with the help of USG abdomen and pelvis and CECT abdomen and pelvis. On retrospective analysis and history taking it was found that 46 patients had history of chronic alcohol intake.

The mean years of alcohol intake were calculated as 14.4 years and the minimum was 2 years and maximum was found to be 34 years. In 3 patients the acute pancreatitis was associated with gall bladder calculus.Further it was found that 47 patients had mild form of pancreatitis and only 3 had severe variety of pancreatitis and all the patients were managed conservatively.

\section{From this study, it is understood that}

\section{Conclusion}

I. There is rapid rise in the incidence of alcohol induced pancreatitis.

II. There is decrease in the incidence of pancreatitis due to gall stones which may be attributed to early diagnosis and treatment of gall stones.

III. There is also a decrease in the severity of the disease. mild forms of pancreatitis occurs more commonly than the severe forms

IV. There is decrease in overall mortality and morbidity due to acute pancreatitis. 


\section{Limitations}

The study population is taken only from patients presenting to Coimbatore medical college hospital, so to check the validity of results it should be checked in varied population. The study does not include the amount of alcohol intake and it simply includes number of years of alcohol intake.

\section{References}

[1]. Fagenholz PJ, Castillo CF, Harris NS, et al. Increasing United States hospital admissions for acute pancreatitis, 19882003. Ann Epidemiol 2007; 17: 491-497.

[2]. Yadav D, Lowenfels AB. Trends in the epidemiology of the first attack of acute pancreatitis: a systematic review. Pancreas 2006; 33: 323-330.

[3]. Australian Institute of Health and Welfare. The burden of disease and injury in Australia 2003. Canberra: AIHW, 2007. (AIHW Cat. No. PHE 82.) http://www.aihw.gov.au/publication-detail/?id=6442467990 (accessed 4 Feb 2015).

[4]. Frey CF, Zhou H, Harvey DJ, White RH. The incidence and case-fatality rates of acute biliary, alcoholic, and idiopathic pancreatitis in California, 1994-2001. Pancreas 2006; 33: 336-344.

[5]. Chen Y, Zak Y, Hernandez-Boussard T, et al. The epidemiology of idiopathic acute pancreatitis, analysis of the nationwide inpatient sample from 1998 to 2007. Pancreas 2013; 42: 1-5.

[6]. Appelros S, Borgström A. Incidence, aetiology and mortality rate of acute pancreatitis over 10 years in a defined urban population in Sweden. Br J Surg 1999; 86: 465-470.

[7]. Banks PA, Bollen TL, Dervenis C, et al; Acute Pancreatitis Classification Working Group. Classification of acute pancreatitis-2012: revision of the Atlanta classification and definitions by international consensus. Gut 2013; 62: 102-111.

[8]. Jacob AO, Stewart P, Jacob O. Early surgical intervention in severe acute pancreatitis: Central Australian experience. ANZ J Surg 2014. 30 May 2014. doi: 10.1111/ans.12707. [Epub ahead of print].

[9]. Tenner S, Baillie J, Dewitt J, et al. American College of Gastroenterology guideline: management of acute pancreatitis. Am J Gastroenterol 2013; 108: 1400-1415.

[10]. Working Group IAP/APA Acute Pancreatitis Guidelines. IAP/APA evidence-based guidelines for the management of acute pancreatitis. Pancreatology 2013; 13(4 Suppl 2): e1-e15.

[11]. Kingsnorth A, O'Reilly D. Acute pancreatitis. BMJ 2006; 332: 1072-1076.

[12]. Barreto SG, Tiong L, Williams R. Drug-induced acute pancreatitis in a cohort of 328 patients. A single-centre experience from Australia. JOP 2011; 12: 581-585.

[13]. Lee JK, Enns R. Review of idiopathic pancreatitis. World J Gastroenterol2007; 13: 6296-6313.

[14]. Ortega AR, Gómez-Rodríguez R, Romero M, et al. Prospective comparison of endoscopic ultrasonography and magnetic resonance cholangiopancreatography in the etiological diagnosis of "idiopathic" acute pancreatitis. Pancreas 2011; 40: 289-294.

[15]. Fleszler F, Friedenberg F, Krevsky B, et al. Abdominal computed tomography prolongs length of stay and is frequently unnecessary in the evaluation of acute pancreatitis. Am J Med Sci 2003; 325: 251-255 